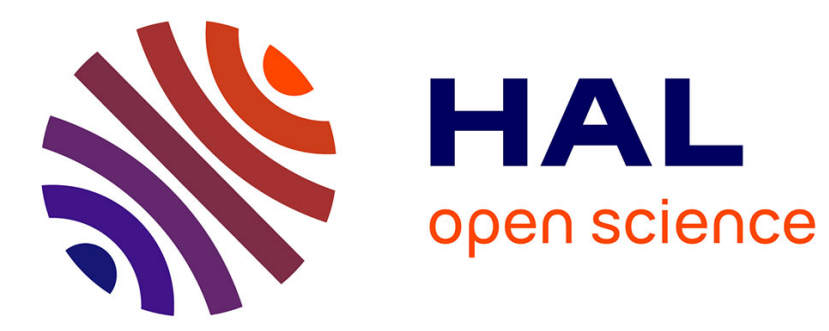

\title{
An atmospheric pressure self-pulsing micro thin-cathode discharge
}

Beilei Du, Sebastian Mohr, Dirk Luggenhölscher, Uwe Czarnetzki

\section{To cite this version:}

Beilei Du, Sebastian Mohr, Dirk Luggenhölscher, Uwe Czarnetzki. An atmospheric pressure selfpulsing micro thin-cathode discharge. Journal of Physics D: Applied Physics, 2011, 44 (12), pp.125204. 10.1088/0022-3727/44/12/125204 . hal-00604888

\section{HAL Id: hal-00604888 \\ https://hal.science/hal-00604888}

Submitted on 30 Jun 2011

HAL is a multi-disciplinary open access archive for the deposit and dissemination of scientific research documents, whether they are published or not. The documents may come from teaching and research institutions in France or abroad, or from public or private research centers.
L'archive ouverte pluridisciplinaire HAL, est destinée au dépôt et à la diffusion de documents scientifiques de niveau recherche, publiés ou non, émanant des établissements d'enseignement et de recherche français ou étrangers, des laboratoires publics ou privés. 


\title{
An atmospheric pressure self-pulsing micro thin cathode discharge
}

\author{
Beilei Du, Sebastian Mohr, Dirk Luggenhölscher and Uwe Czarnetzki \\ Institute for Plasma and Atomic Physics, Ruhr-University Bochum, 44780 Bochum, \\ Germany \\ E-mail: Beilei.Du@ep5.rub.de
}

\begin{abstract}
A micro thin cathode discharge ( $\mu \mathrm{TCD}$ ) is a modified micro hollow cathode discharge (MHCD) where the anode is much thicker than the one in a MHCD, which allows a stable operation at atmospheric pressure. The $\mu \mathrm{TCD}$ was operated at different pressures in argon by a DC voltage. At atmospheric pressure, the $\mu$ TCD operates in a self-pulsing mode with current peaks with a duration of a few nanoseconds and a current density up to $10^{5} \mathrm{~A} / \mathrm{cm}^{2}$, supplied by the charge stored in the capacitance of the discharge device. The plasma emission was observed by an ICCD camera. Both, the voltage - current characteristic and the ICCD image indicate that this self-pulsing is due to a periodic ignition of a spark discharge. The electron density was measured by the Stark broadening of the $\mathrm{H}_{\beta}$-line with an admixture of $1 \%$ hydrogen. In the periodic spark mode the electron density is of the order of $10^{16} \mathrm{~cm}^{-3}$. The direct measurement of the discharge current in these kind of discharges is not possible, since it is driven by the inherent capacitance of the discharge device. Therefore, an electrical model including stray capacitances is set up. The modelled results agree very well with the measured currents and voltages.
\end{abstract}

PACS numbers: 52.25.Os, 52.50.Dg, 52.50.Nr, 52.70.Ds, 52.70.Kz, 52.80.Mg 


\section{Introduction}

As a kind of microcavity discharge, micro hollow cathode discharges have simple electrode geometries [1] and have found various applications in lighting as UV radiation sources [2 - 6], as plasma cathodes to generate large volumes of atmospheric pressure plasmas [7] as well as for analytical applications [8]. Basically, a MHCD consist of two electrode layers (anode and cathode) separated by a dielectric layer. Each layer is several $100 \mu \mathrm{m}$ thick. The discharge is developed in a hole (several $100 \mu \mathrm{m}$ in diameter) which penetrates the cathode and the dielectric. The anode layer can have various shapes [9]. In the current research, the common anode shape is identical to the cathode $[8,10-12]$.

Under certain conditions, the MHCD shows self-pulsing with oscillations of the discharge voltage and current [9 - 12]. At medium pressure, several tens of hectopascal, the MHCD can be operated in a selfpulsing mode with a current peak of several milliamperes and with a duration of several microseconds. Rousseau and coworkers [12] showed that the self-pulsing is related to a periodic transition between two modes: the plasma is confined inside the hole and the plasma expands outside the hole onto the cathode backside. On the other hand, the MHCD can be operated in another self-pulsing mode with a current peak which is much higher than several milliamperes and with a duration of only several nanoseconds. This self-pulsing mode was reported by Schoenbach [9] firstly in a MHCD with a closed anode at over-atmospheric pressure. In our study, we chose an anode with the same hole diameter as the one of the cathode, but which is much thicker $(1 \mathrm{~mm})$ than the cathode and the dielectric, since the thick anode provides the mechanical stability for the whole discharge setup.

The time resolved discharge voltage and current measurement showed that our discharge operates in both self-pulsing modes. In our study, we focus on the self-pulsing with high current peaks with nanosecond a duration at atmospheric pressure. The plasma current density and the ICCD image of the plasma volume indicate that this self-pulsing mode is related to a periodically repeated ignition of a spark discharge. The thinness of the cathode (in our case $100 \mu \mathrm{m}$ ) leads to enable the cathode to heat very quickly and to emit electrons by thermionic emission with a high current density. Although we have no detailed measurements on the hypothesis of a hot cathode spot, reversal of the polarity of the applied voltage inhibits the development into the self-pulsing regime. Thus, we prefer to call this device micro thin cathode discharge ( $\mu \mathrm{TCD}$ ) instead of micro hollow cathode discharge. The electron density in argon was measured by the Stark broadening of the $\mathrm{H}_{\beta}$-line with an admixture of $1 \%$ hydrogen. The time averaged electron density can reach up to $10^{16} \mathrm{~cm}^{-3}$. In the voltage and current measurement, a peak current as high as several amperes was measured. Due to a $500 \mathrm{k} \Omega$ series resistor, the peak current measured on the anode side should be in the milliampere range. An electrical model was built and carefully analyzed. The existence of a stray capacitance is the reason for the measured high peal current. Very good agreement between the modelling results and the measurement was obtained. 
The construction of the $\mu \mathrm{TCD}$ and the experimental setup are introduced in the next chapter, followed by the measurement results including the analyses in chapter 3 . Finally, conclusions are drawn.

\section{Experimental setup}

Because of the expected high gas temperature of about $1000 \mathrm{~K}$ [13], molybdenum and alumina were used as electrodes and dielectric layer respectively. Different from the usual MHCD with symmetric electrodes, the electrodes of the $\mu \mathrm{TCD}$ are asymmetric (Figure 1a). One electrode, which was used as anode, is much larger and thicker than the other one. The dielectric layer has the same thickness as the cathode and separates the electrodes. To avoid an unwanted electrode edge ignition, the dielectric is a little larger than the anode. The plasma is developed in a mechanically drilled hole with a diameter of $200 \mu \mathrm{m}$ penetrating the sandwich structure. The three layers are pressed together by a PVC holder fixed by screws (Figure 1b). The thick and large anode provides mechanical stability for the fragile alumina layer. The holder has an axial opening with $5 \mathrm{~mm}$ diameter in order to avoid obstructing the plasma development on the electrode surface. The capacitance of the entire discharge setup including the PVC holder is $120 \mathrm{pF}$, measured by a LCR meter.
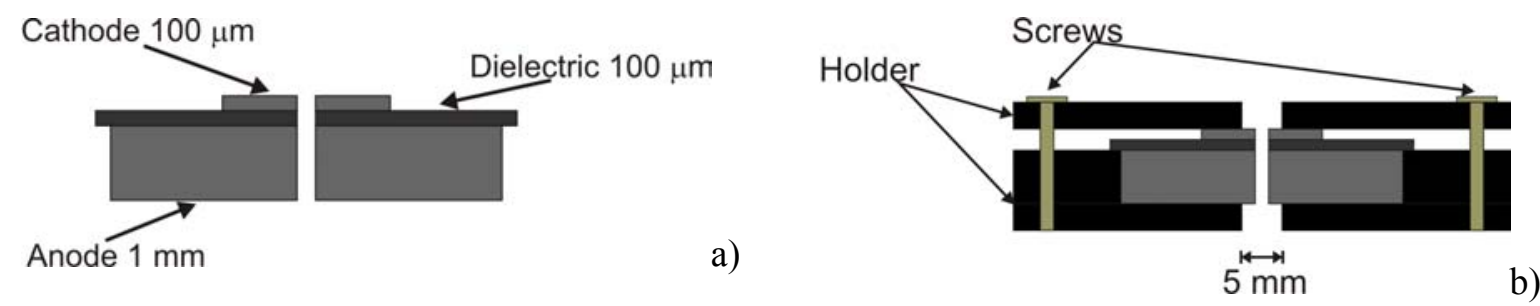

Figure 1. Cross section of the $\mu \mathrm{TCD}$ electrode geometry (a) and with the holder (b).

The discharge is generated by a DC voltage supply. To avoid an overheating of the electrodes, the applied voltage was pulsed with $10 \mathrm{~Hz}$ and with $1 \%$ duty cycle. A $500 \mathrm{k} \Omega$ series resistor $R_{l}$ and a 100 $\Omega$ measurement resistor $R_{2}$ were connected in series at the cathode side and the anode side respectively (Figure 2). High voltage probes (Lecroy PPE20kV) were used to measure the discharge voltage and the current via the measurement resistor $R_{2}$. A fast oscilloscope (Lecroy Wavepro 7100A) was used to read out the temporally resolved potentials, $V_{l}$ and $V_{2}$. The discharge voltage $U_{d}$ and the current $I_{R}$ via $R_{2}$, are deduced from the measured potentials:

$$
\begin{gathered}
U_{d}=V_{1}-V_{2}, \\
I_{R}=\frac{V_{2}}{R_{2}} .
\end{gathered}
$$

In a DC discharge, $I_{R}$ equals the plasma current. When the discharge operates in the self-pulsing mode, according to the experimental setup, the AC part of the plasma current $I_{p l}$ cannot be directly measured on $R_{2}$ but can be deduced from the change of the discharge voltage in case that the external current measured on $R_{2}$ is much smaller than the discharge current inside the capacitor: 


$$
I_{p l} \approx I_{d}=C_{d} \frac{d U_{d}}{d t},
$$

where $C_{d}$ is the capacitance of $\mu \mathrm{TCD}$ device.

The emission spectrum of the $\mu \mathrm{TCD}$ was recorded by a $2 \mathrm{~m}$-spectrograph with $0.01 \mathrm{~nm}$ spectral resolution equipped with an intensified diode array detector. The gate-width is $33 \mathrm{~ms}$ which is longer than the duration of the applied voltage. Therefore, the emission is time integrated. An optical fibre mounted at the cathode side guides the emission light to the spectrograph, i.e. the emission spectrum is spatially integrated, too.

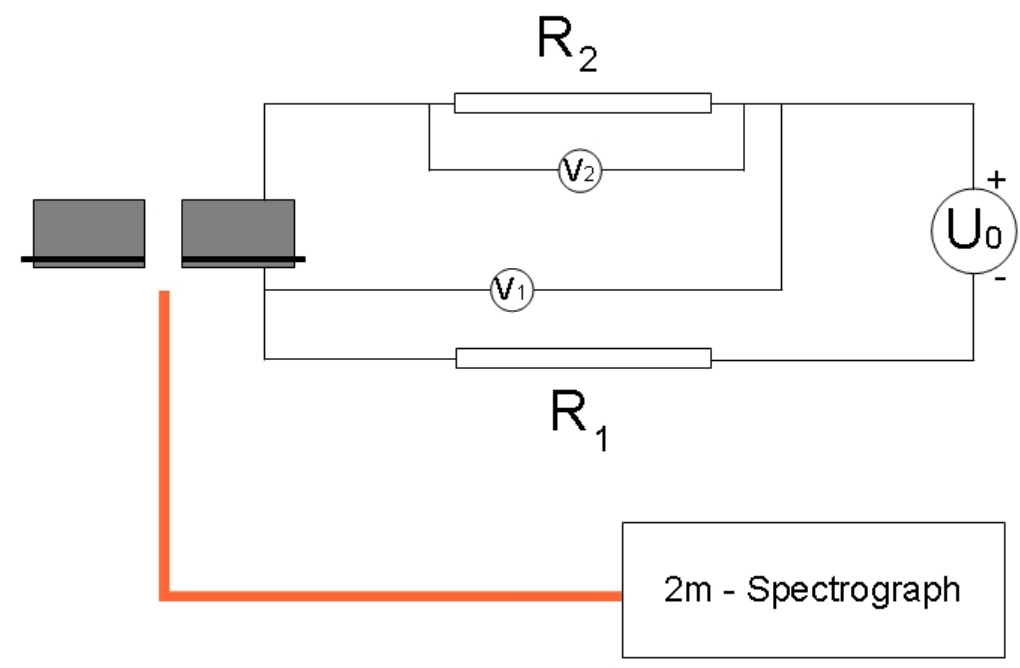

Figure 2. Experimental setup.

The $\mu \mathrm{TCD}$ was placed in a vacuum chamber and operated in argon with $100 \mathrm{sccm}$ gas flow. The gas pressure ranges from several tens of hectopascal up to atmospheric pressure. For electron density measurements, $1 \%$ hydrogen was admixed. The electron density is determined by the Stark broadening of the $\mathrm{H}_{\beta}$-line (486.3 nm) [14 - 17].

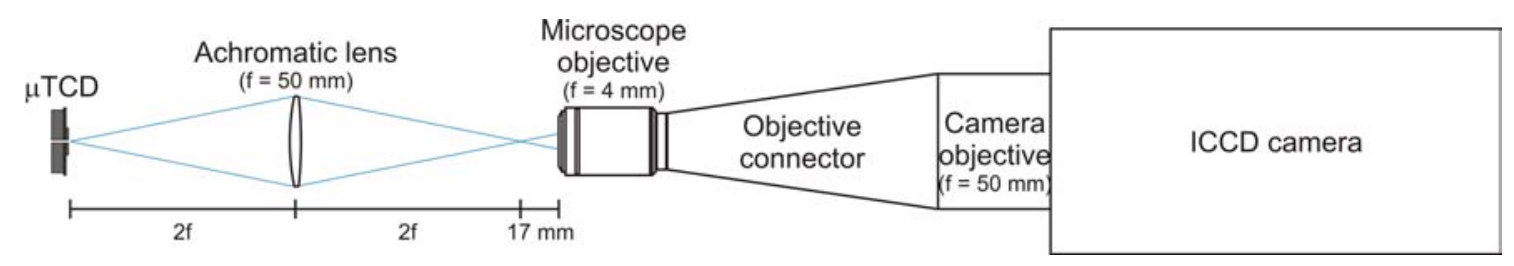

Figure 3. Concept for imaging the plasma volume.

In order to obtain the emission image of the plasma volume, a microscope objective with $17 \mathrm{~mm}$ working distance (Nikon CFI L Plan Epi SLWD 50X) was mounted in front of the camera objective (Figure 3). In this way, the image is magnified. The magnification depends on the focal length of both objectives. The focal lengths of the microscope objective $f_{m}$ and the camera objective $f_{c}$ are $4 \mathrm{~mm}$ and $50 \mathrm{~mm}$ respectively. The magnification is then: 


$$
M=\frac{f_{c}}{f_{m}}=12.5
$$

Because of the limitation of the vacuum chamber, the $\mu \mathrm{TCD}$ cannot be placed in the working distance of the microscope objective. An achromatic lens with $50 \mathrm{~mm}$ focal length was used to image the plasma volume outside the vacuum chamber in a ratio of $1: 1$. This image is magnified by the objective system and recorded.

\section{Results and discussion}

\subsection{Two self-pulsing modes}

\subsubsection{Self-pulsing at atmospheric pressure}

At atmospheric pressure temporally resolved voltage and current measurements showed that the $\mu \mathrm{TCD}$ operates in a self-pulsing mode with a very stable frequency (Figure 4a). Zooming in Figure 4a, the voltage drops very fast to zero (Figure $4 \mathrm{~b}$ ). When the voltage drops, the plasma current $I_{p l}$ reaches a peak value as high as $30 \mathrm{~A}$, and lasts only several nanoseconds. According to the discharge cross section, the current density is as high as $10^{5} \mathrm{~A} / \mathrm{cm}^{2}$. The current $I_{R}$ via the resistor $R_{2}$ is only $10 \%$ of the plasma current, but much higher than the limit due to the series resistor. The current $I_{R}$ will be discussed in section 3.3 along with an electrical model. Figure $4 \mathrm{c}$ shows the time integrated ICCD image of the $\mu \mathrm{TCD}$ cathode side. Hot electrons are located near the edge of the cathode hole, generating the emission. Both the high current density and the distribution of the hot electrons indicate a periodic thermionic emission, accompanied by the voltage and current oscillation. At atmospheric pressure the self-pulsing mode is a periodically repeated ignition of a spark discharge due to the thermionic emission. The thermionic emission refers to the phenomenon of electron emission from a high-temperature cathode surface. 


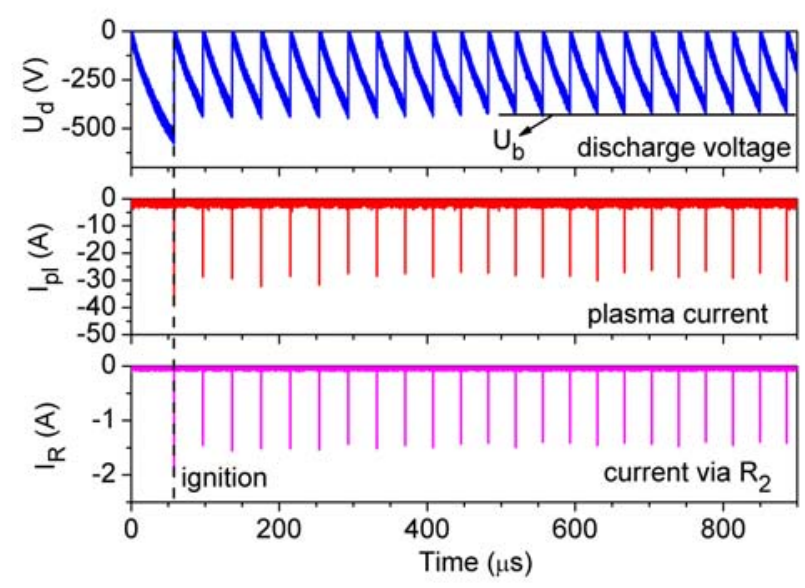

a)

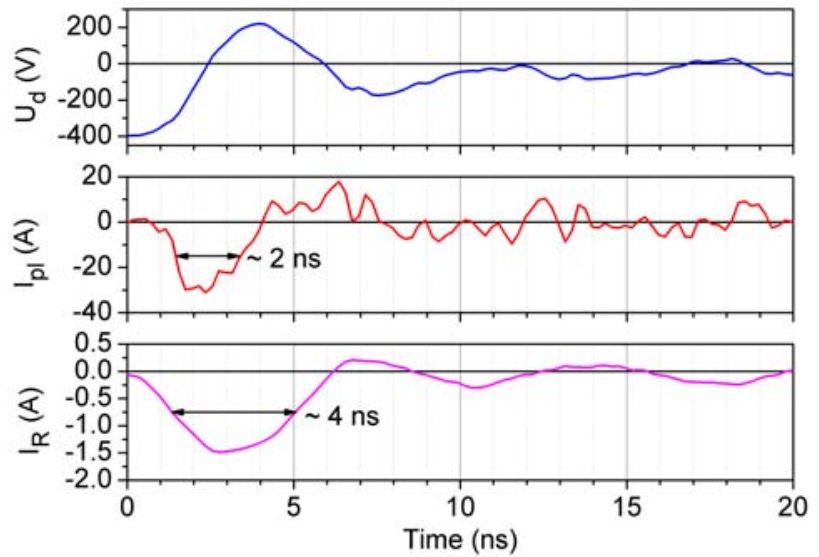

b)

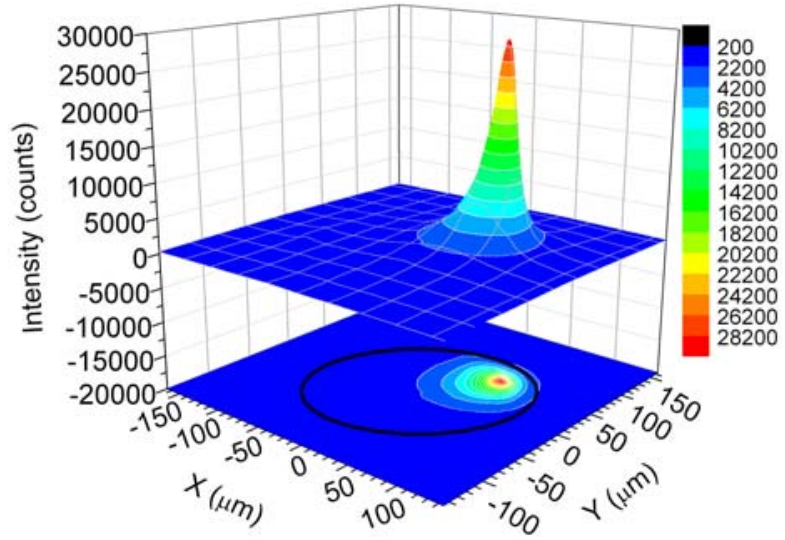

c)

Figure 4. a) The temporally resolved discharge voltage and the currents at $950 \mathrm{hPa}$ with $1000 \mathrm{~V}$ applied voltage; b) Zoom of one single current peak; c) ICCD image of $\mu$ TCD at $1000 \mathrm{hPa}$ with $900 \mathrm{~V}$ applied voltage. The black circle in the projection indicates the orifice of the discharge hole. 

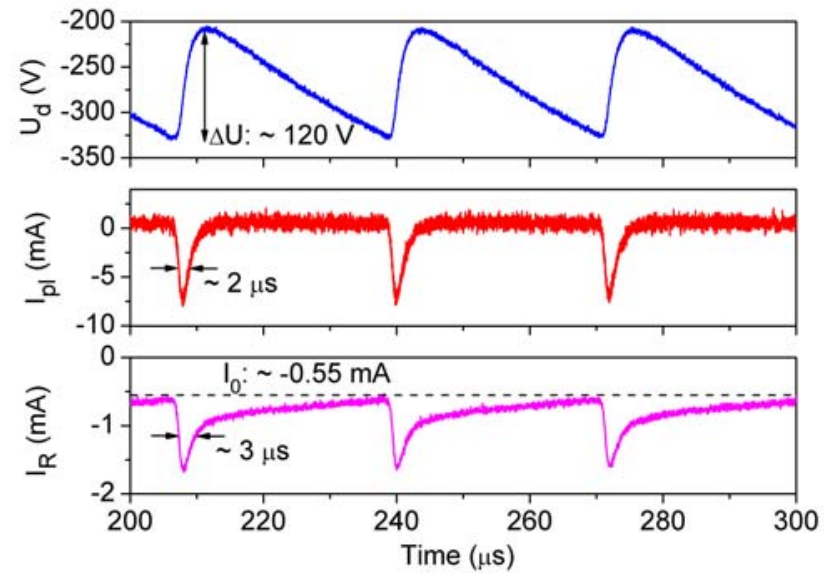

a)

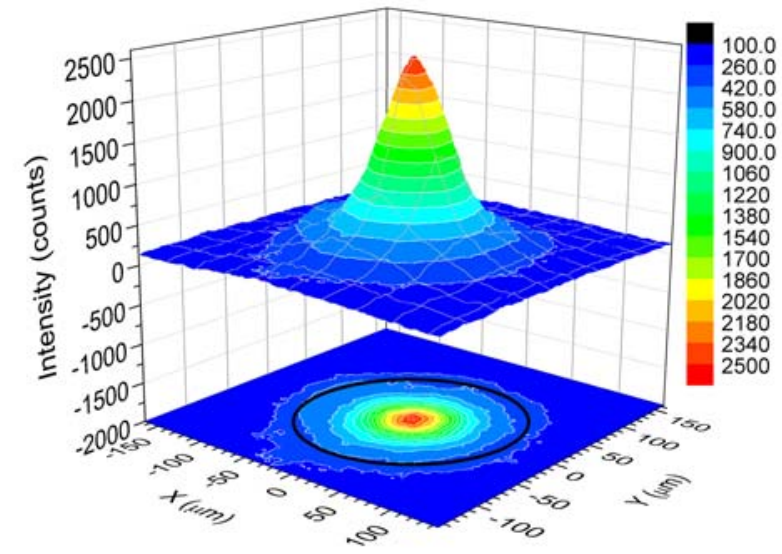

b)

Figure 5. a) Temporally resolved discharge voltage and current of the $\mu \mathrm{TCD}$ at $50 \mathrm{hPa}$ with $600 \mathrm{~V}$ applied voltage; b) ICCD image of $\mu \mathrm{TCD}$ at $60 \mathrm{hPa}$ with $600 \mathrm{~V}$ applied voltage. The black circle in the projection indicates the orifice of the discharge hole.

\subsubsection{Self-pulsing at medium pressure}

At medium pressure the $\mu \mathrm{TCD}$ shows a self-pulsing similar to the self-pulsing in a MHCD. The voltage oscillates but does not drop to zero. The peak current is in the range of milliamperes with a duration of a few microseconds (Figure 5a), which is obviously longer than the current peak width in the self-pulsing at atmospheric pressure showed in Figure 4b. This self-pulsing is explained by Rousseau and coworkers $[11,12]$. The oscillation of the discharge voltage and current is related to a periodic transition between two modes: the plasma is confined inside the hole and the plasma expands outside the hole onto the cathode backside. The time integrated ICCD image of the $\mu$ TCD shows that the hot electrons are located in the centre of the hole (Figure 5b). Although the emission during the expansion onto the cathode backside surface is much weaker than the emission from the plasma confined inside the hole, one can still recognize this mode in Figure $5 \mathrm{~b}$.

The exposure times for the images in Figure $4 \mathrm{c}$ and Figure $5 \mathrm{c}$ are both $1 \mathrm{~ms}$ which is the duration of the applied voltage. The self-pulsing frequencies for both cases are about $25 \mathrm{kHz}$ which means that each exposure integrates over about 25 pulses. However, the duration of each single pulse in the case 
of the spark mode is much shorter (several nanoseconds) than in the other case. Therefore, the integrated maximum intensity for the spark mode is only one order of magnitude higher. The discharge is always constricted at the edge when it goes into the spark mode. At pressures close to the threshold, the transition to this mode can be stochastic. Although the location at the edge of the discharge in the spark mode can vary, it is usually fixed over a large number of pulses.

The $\mu$ TCD shows two self-pulsing modes in different pressure regions. At medium pressure the selfpulsing is due to the plasma expansion which is the same as in a MHCD, although the $\mu$ TCD anode is much thicker than the one of the MHCD. At atmospheric pressure the self-pulsing discharge produces strongly localized sparks. In this case, the thinness of the cathode is more important than its hollow structure.

\subsection{Electron density}

The electron density was measured by the Stark broadening of the $\mathrm{H}_{\beta}$-line. Figure 6 shows the time averaged recorded $\mathrm{H}_{\beta}$-line at $900 \mathrm{hPa}$ with $1.2 \mathrm{kV}$ applied voltage. Besides the Stark broadening and the instrumental broadening, the Doppler broadening, the natural broadening, the resonance broadening and the van der Waals broadening also contribute to the line profile. Considering the experimental conditions, the natural broadening and the resonance broadening are negligible compared to the other broadening effects.

The FWHM of the Doppler broadening profile is determined by the following expression [18]:

$$
\Delta \lambda_{\text {Doppler }}=7.715 \cdot 10^{-5} \lambda[\mathrm{nm}] \sqrt{\frac{k_{B} T[\mathrm{eV}]}{m[u]}},
$$

where $\lambda$ is the wavelength, $T$ is the gas temperature and $m$ is the atomic weight of the hydrogen atom. The dipole interaction of an excited atom with the induced dipole of a ground state atom results in the van der Waals broadening. The FWHM of the van der Waals broadening profile in approximated by the following expression which is strictly applicable to hydrogen and similar atomic structures [19]:

$$
\Delta \lambda \approx 3 \cdot 10^{-18} \lambda^{2}[\mathrm{~nm}]^{2} C_{6}^{2 / 5}\left(\frac{T[K]}{\mu[u]}\right)^{3 / 10} N\left[\mathrm{~cm}^{-3}\right],
$$

where $\lambda$ is the wavelength, $T$ is the gas temperature, $\mu$ is the reduced mass of the atom-perturber (argon) and $N$ is the perturber density. $C_{6}$ is the interaction constant and is calculated according to [17]. In case of this experiment, the interaction constant is $3 \cdot 10^{-31}$.

The gas temperature is estimated to be $500 \mathrm{~K}$ at medium pressure and $1000 \mathrm{~K}$ at atmospheric pressure [8]. With these gas temperatures, the FWHMs of both Doppler broadening and van der Waals broadening contribute less than $10 \%$ to the line profile. Therefore, the contribution of Doppler broadening and van der Waals broadening was neglected in our measurement.

The interaction of the emitter atoms with charged particles results in the Stark broadening. The FWHM of the Stark broadening profile is approximated by following relation [20]: 


$$
\frac{\Delta \lambda}{[\mathrm{nm}]}=4.8 \cdot\left(\frac{n_{e}}{10^{17}\left[\mathrm{~cm}^{-3}\right]}\right)^{0.68116}
$$

Due to the low $\mathrm{H}_{\beta}$ emission intensity, the entrance slit of the spectrograph is widely opened. This leads to the instrumental broadening with a Gaussian profile with a FWHM of $0.12 \mathrm{~nm}$, which is not negligible. A Voigt function is fitted to the measurement profile (Figure 6). The electron density is obtained from the remaining Lorentzian part of the Voigt profile. This does not include uncertainties resulting from spatial integration in the measurement and the applied line broadening model in the analysis. Both uncertainties are difficult to quantify. Figure 7 shows the time and spatially integrated electron density and the plasma current at different pressures with $1.2 \mathrm{kV}$ applied voltage. Regarding the dependency between the electron density and the gas pressure, Figure 7 can be divided into two regions. Region 1: the electron density increases linearly with the pressure. In this region, the temporally resolved voltage and current show neither oscillation, as showed in Figure 5a, nor the periodic sparks. The discharge operates in DC mode with plasma current of several milliamperes; Region 2: the electron density increases to the order of $10^{16} \mathrm{~cm}^{-3}$ and stays constant. In this region, the $\mu \mathrm{TCD}$ shows the self-pulsing due to the periodic ignition of the spark discharge, where the peak currents are several tens of amperes. It should be mentioned that the plasma currents are measured in pure Ar discharges, where the discharge operates in the periodic spark mode above $600 \mathrm{hPa}$. The pressure where the transition between region 1 and region 2 occurs depends on the gas composition and the applied voltage, and varies between $300 \mathrm{hPa}$ and $700 \mathrm{hPa}$.

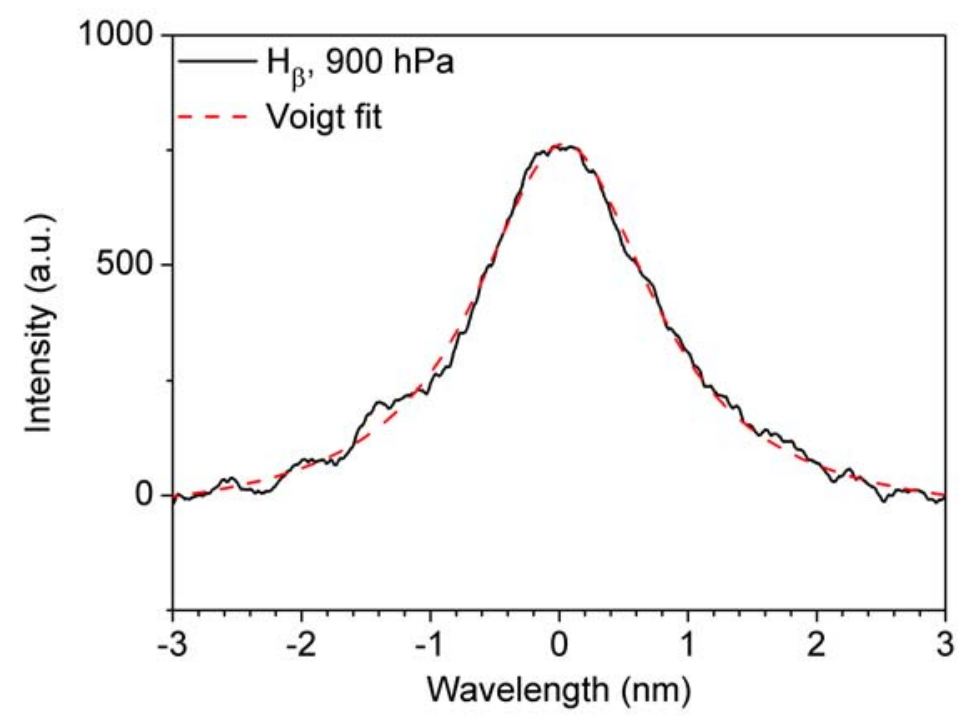

Figure 6. Solid line: the recorded $\mathrm{H}_{\beta}$-line at $900 \mathrm{hPa}$ with $1.2 \mathrm{kV}$ applied voltage; dashed line: Voigt fit with consideration of the instrumental broadening. 


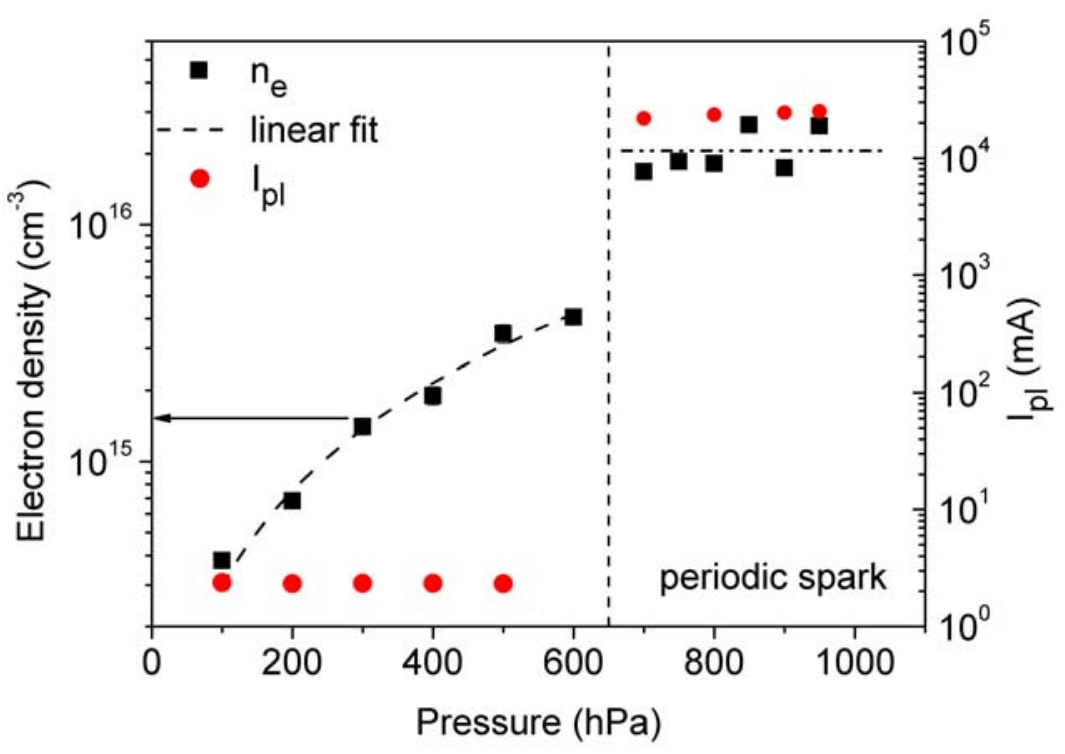

Figure 7. Average electron density and the plasma current in the $\mu \mathrm{TCD}$ with $1.2 \mathrm{kV}$ applied voltage at various pressures. The plasma currents are measured in the pure Ar discharges. The measurement uncertainty of the electron density is about the size of the symbols.

\subsection{Discussion of the measured current}

The $\mu$ TCD self-pulsing mode (the periodic spark mode) in the pure argon discharge appears at pressures from several $300 \mathrm{hPa}$ up to atmospheric pressure with frequencies ranging from several kilohertz up to several tens of kilohertz. With the same applied voltage, the self-pulsing frequency decreases with the increasing of the pressure, while the breakdown voltage increase (Figure 8a). Further, Figure $8 \mathrm{~b}$ shows that the self-pulsing frequency at different pressures depends on the ratio of the applied voltage $U_{0}$ to the self-pulsing breakdown voltage $U_{b}$ as indicated in Figure 4a. In order to explain this behaviour, an equivalent circuit is made (Figure 9) with a plasma resistance $R_{p l}$ parallel to the $\mu \mathrm{TCD}$ setup capacitance $C_{d}$. This model is also used by Rousseau and coworkers [12] to describe the self-pulsing at medium pressure. Graves et al [21] used a more elaborate model that includes also the differential resistance which is approximated by a third order polynomial. However, in their case the discharge oscillates between the Townsend, normal, and abnormal glow regimes. In our case of the self-pulsing at high pressure, transition is made to the high current spark regime, characterized by a negative differential resistance. The corresponding instability is terminated by the finite charge in the capacitor that limits the discharge time to only a few nanoseconds. As a first and simple approach here only a fixed plasma resistance is assumed. This allows already description of some of the major characteristics. More elaborate models are desirable including also thermionic emission from the surface. However, this would go beyond the scope of this work.

Following the equivalent circuit in Figure 9, the discharge voltage $U_{d}$ can be deduced as follows:

$$
U_{d}=U_{0}\left(1-e^{-t / R_{1} \cdot C_{d}}\right) \text {. }
$$


At $t=t_{b}$, the discharge voltage $U_{d}$ reaches the breakdown voltage $U_{b}$, then Equation 4 can be written in following expression with $f=1 / t_{b}$ :

$$
f=\left(-R_{1} C_{d} \cdot \ln \left(1-\frac{U_{b}}{U_{0}}\right)\right)^{-1} .
$$

Adjusting the $R_{l} C_{d}$ parameter in Equation 5 until the calculated curve fits the measured profile (Figure 8), the $\mu \mathrm{TCD}$ setup capacitance $C_{d}$ is determined. The capacitance $C_{d}$ is about $130 \mathrm{pF}, 10 \mathrm{pF}$ above the value measured by the LCR meter. This $10 \mathrm{pF}$ can be contributed by the stray capacitances originating from coaxial cables in the experimental setup. With this assumption, the equivalent circuit was modified by a stray capacitance $C_{s}$ parallel to the $\mu \mathrm{TCD}$ capacitance $C_{d}$ (Figure 10). The existence of the stray capacitance is also discussed about in [12]. During the charging process, the discharge voltage increases, the current $I_{R}$ is the charging current of the $\mu$ TCD capacitance (Figure 10a). Once the discharge voltage reaches the breakdown voltage, the discharging process begins (Figure 10b). The discharge voltage drops to zero. The discharging current of the stray capacitance contributes to the current $I_{R}$. Therefore, the measured value of $I_{R}$ is much higher than it should be due to the limit by the series resistor $R_{l}$. 


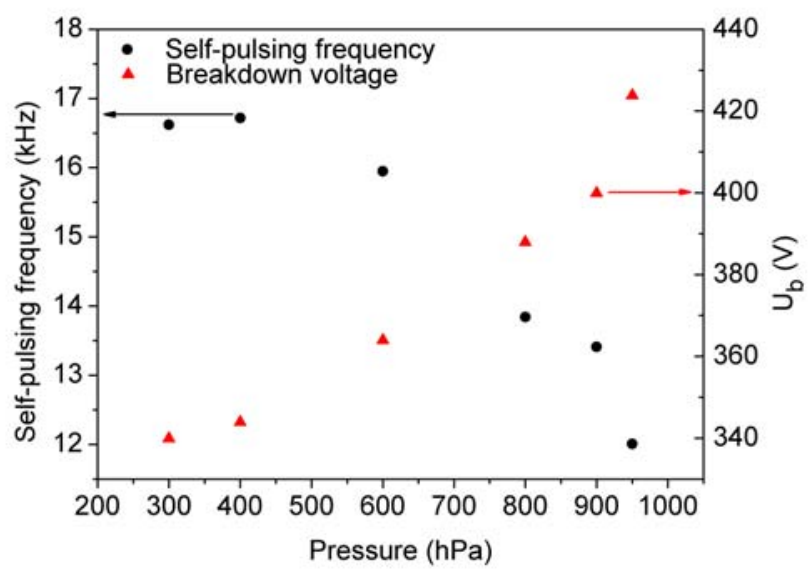

a)

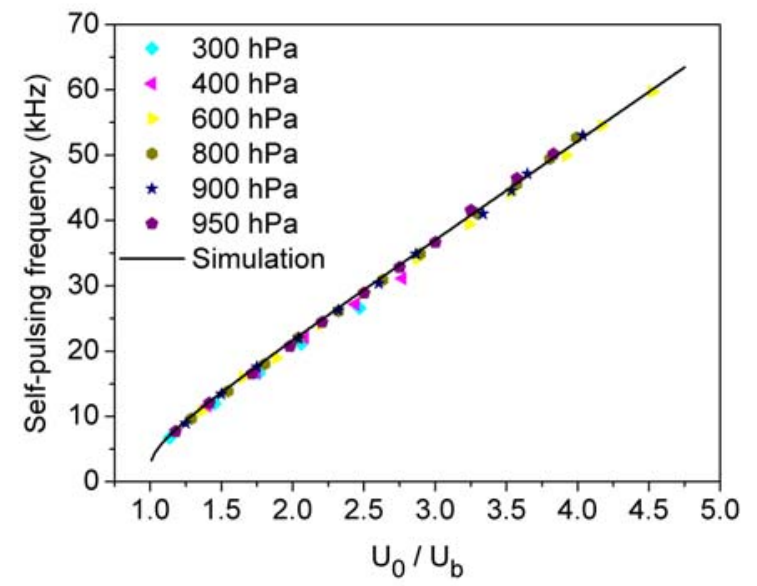

b)

Figure 8. a) The self-pulsing frequency and the breakdown voltage in the $\mu \mathrm{TCD}$ with $600 \mathrm{~V}$ applied voltage at various pressures. b) The self-pulsing frequency of the $\mu$ TCD depends on the ratio of the applied voltage $U_{0}$ to the breakdown voltage $U_{b}$. The breakdown voltage depends on the gas pressure and the dimension of the plasma volume.

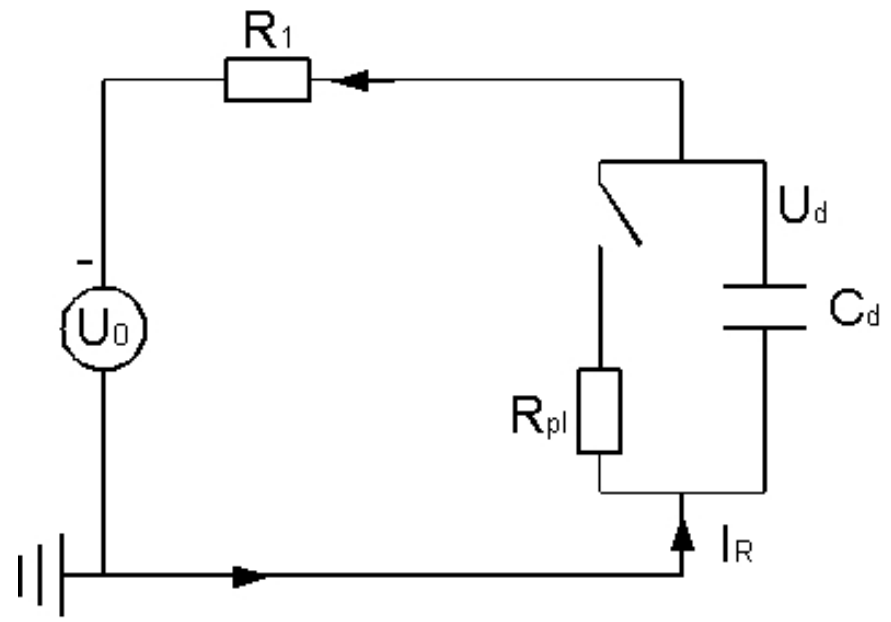

Figure 9. The equivalent circuit. The measurement resistance $R_{2}$ is neglected. 


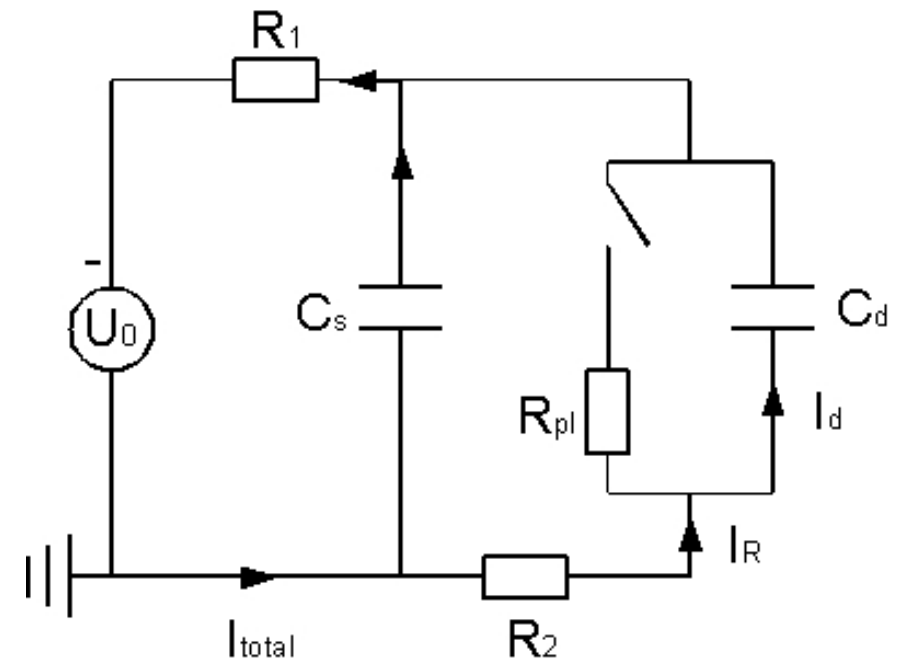

a)

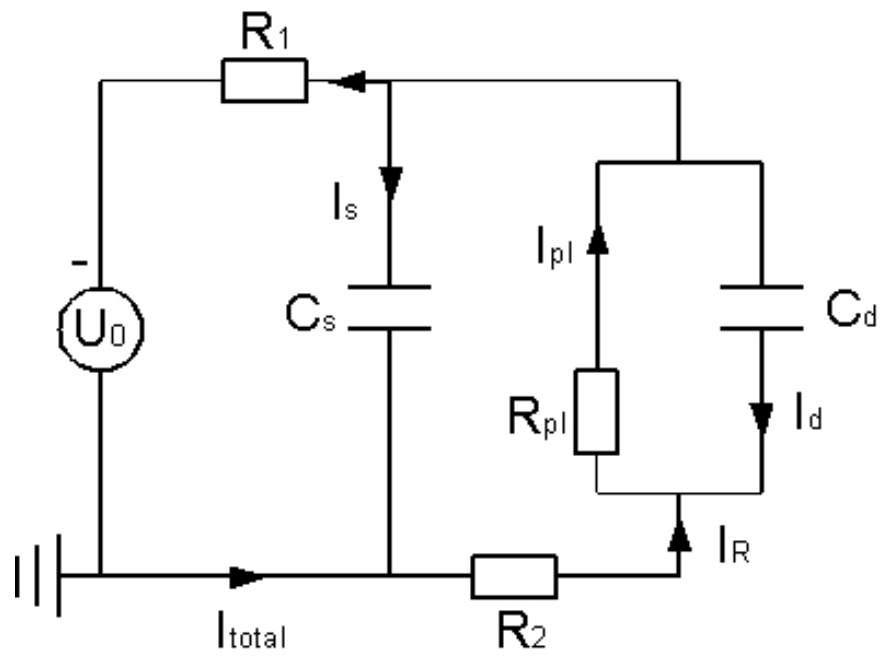

b)

Figure 10. Modified equivalent circuit with stray capacitance: a) charging process; b) discharging process.

Following the modified equivalent circuit (Figure 10), the charging and the discharging processes are described by two differential equations.

Charging process:

$$
\ddot{I}_{\text {total }}+\left(\frac{1}{R_{2} C_{d}}+\frac{1}{R_{1} C_{s}}+\frac{1}{R_{2} C_{s}}\right) \dot{I}_{\text {total }}+\frac{1}{R_{1} C_{d} \cdot R_{2} C_{s}} I_{\text {total }}=0 ;
$$

Discharging process:

$$
\ddot{I}_{p l}+\left(\frac{1}{R_{p l} C_{d}}+\frac{1}{R_{2} C_{d}}+\frac{\left(1+R_{2} / R_{1}\right)}{R_{2} C_{s}}\right) \dot{I}_{p l}+\frac{1}{R_{2} C_{s} \cdot R_{p l} C_{d}}\left(1+\frac{R_{2}}{R_{1}}+\frac{R_{p l}}{R_{1}}\right) I_{p l}=\frac{U_{0}}{R_{1}} \cdot \frac{1}{R_{2} C_{s} \cdot R_{p l} C_{d}} .
$$


The differential equation for the charging process (Equation 6) is easily solved with initial conditions $I_{\text {total }}(t=0)=\frac{U_{0}}{R_{1}}$ and $\dot{I}_{\text {total }}(t=0)=-\frac{U_{0}}{R_{1}^{2} \cdot C_{s}}$, where $C_{s}=10 \mathrm{pF}$, as obtained above. The current $I_{R}$ and the discharge voltage $U_{d}$ are deduced:

$$
\begin{gathered}
I_{R}=I_{\text {total }}+R_{1} C_{s} \dot{I}_{\text {total }}, \\
U_{d}=U_{0}-I_{\text {total }} R_{1}-I_{R} R_{2} .
\end{gathered}
$$

During the charging process the plasma current is zero. When the discharge voltage $U_{d}$ reaches the breakdown voltage $U_{b}$, the discharging process begins. With initial conditions $I_{p l}\left(t=t_{b}\right)=\frac{U_{b}}{R_{p l}}$ and $\dot{I}_{p l}\left(t=t_{b}\right)=\frac{U_{0}-U_{b}}{R_{1}+R_{2}}-\frac{U_{b}}{R_{p l}}$, the plasma current $I_{p l}$ is obtained by solving Equation 7. The current $I_{R}$ and the discharge voltage $U_{d}$ are deduced as follows:

$$
\begin{gathered}
I_{R}=I_{p l}+R_{p l} C_{d} \dot{I}_{p l}, \\
U_{d}=R_{p l} \cdot I_{p l} .
\end{gathered}
$$

The quantities of the parameters in the upper differential equations except the plasma resistance $R_{p l}$ are given in Table 1.

Table 1. Parameters used in Equation 6 and 7.

\begin{tabular}{|c|c|c|}
\hline Applied voltage & $U_{0}$ & $-1000 \mathrm{~V}$ \\
\hline Breakdown voltage & $U_{c}$ & $-440 \mathrm{~V}$ \\
\hline Series resistance & $R_{l}$ & $500 \mathrm{k} \Omega$ \\
\hline Measurement resistance & $R_{2}$ & $100 \Omega$ \\
\hline$\mu \mathrm{TCD}$ capacitance & $C_{d}$ & $120 \mathrm{pF}$ \\
\hline Stray capacitance & $C_{s}$ & $10 \mathrm{pF}$ \\
\hline
\end{tabular}

Adjusting the value of the plasma resistance $R_{p l}$, one can obtain the best agreement between the measured and simulated currents when $R_{p l}$ is $14.5 \Omega$. The simulation results are shown in Figure 11 . Once the discharge voltage reaches the breakdown voltage, the discharge is ignited (Figure 11a): The discharge voltage drops to zero immediately. At the same time, the plasma current $I_{p l}$ reaches $30 \mathrm{~A}$, and the current $I_{R}$ is $1 \mathrm{~A}$. Quantitatively, the measured (Figure 4a) and the simulated peak currents agree with each other excellently. The good agreement between the measurement results and the simulation results confirms the existence of the stray capacitance. 
The measured temporal development of the discharge voltage and current $I_{R}$ is of the order of the temporal resolution of the high voltage probe. Therefore, it is possible that the development of the discharge voltage and current $I_{R}$ is probably faster. However, with the assumption of the plasma resistance $R_{p l}$, one can obtain a good agreement about the current durations between the simulation (Figure 11b) and the measurement (Figure 4b). Both, the measurement and the simulation show that the plasma current duration is shorter than the current $I_{R}$, which is basically the discharging current of the stray capacitance.

On the other hand, the plasma resistance $R_{p l}$ is connected to the plasma conductivity $\sigma$.

$$
R_{p l}=\frac{l_{p l}}{\sigma \cdot A_{p l}},
$$

where $A_{p l}$ and $l_{p l}$ are the cross section area and the length of the plasma volume. $A_{p l}$ is the full cross section of the hole; $l_{p l}$ is assumed to be $200 \mu \mathrm{m}$. Further, the plasma conductivity is proportional to the electron density $n_{e}: \sigma=\frac{e^{2} \cdot n_{e}}{m_{e} \cdot v}$, where $e$ is the elementary charge, $m_{e}$ is the mass of the electron, $v=$ $4 \cdot 10^{12} \mathrm{~s}^{-1}$ is the collision frequency [22]. Thus, the electron density can be deduced with assumptions of the plasma volume and the plasma resistance, which is obtained from the fitting procedure described before. The deduced electron density is $6.3 \cdot 10^{16} \mathrm{~cm}^{-3}$, three times more than the measured density. One should notice that the electron density was not measured in pure argon, but with an admixture of $1 \%$ hydrogen. Due to this amount of hydrogen, the electron density is lower than the density in the pure argon discharge. Therefore, the discrepancy of factor 3 is reasonable. However, it should be pointed out that the determination of electron density from the fitted plasma parameter is certainly not a very precise method since it is based on a series of simplifying assumptions. Nevertheless, the rather good agreement is a strong indication that the order of magnitude is correct and that the rapid discharging of the capacitor is due to the formation of a rather dense plasma on a nanosecond-scale. 

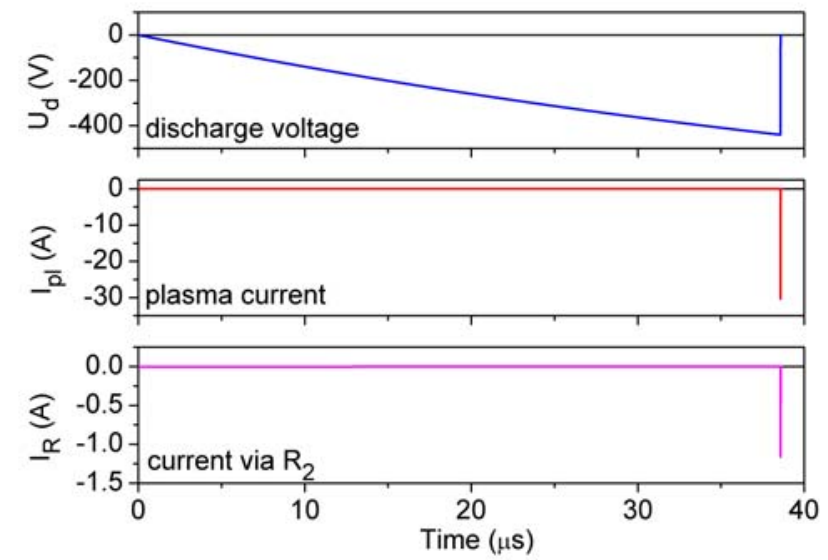

a)
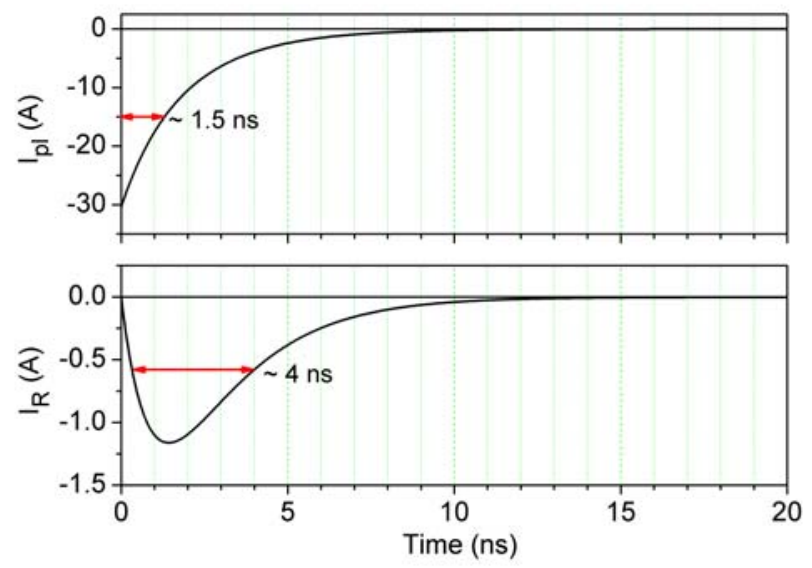

b)

Figure 11. Simulation results $(950 \mathrm{hPa}, 1000 \mathrm{~V})$ : a) The temporally resolved discharge voltage and currents; b) Zoom of one single current peak.

\section{Conclusions}

The micro thin cathode discharge shows two self-pulsing modes: 1) at medium pressure, similar to a micro hollow cathode discharge, related to the periodic transition between plasma expansion on the cathode backside and collapse back inside the hole; 2) at higher pressure up to atmospheric pressure, thermionic electron emission occurs on the thin cathode hole edge. The $\mu$ TCD repeats charging and igniting. A spark discharge is characterized by the discharge voltage and current oscillation as well as the ICCD image. In the periodic spark mode, the electron density in argon was measured to be as high as $10^{16} \mathrm{~cm}^{-3}$ by the Stark broadening of the $\mathrm{H}_{\beta}$-line. Both the measurement and the analysis of an equivalent circuit proved the existence of stray capacitances in the experimental setup, which results in the measured high current peak. The modelled temporal development of discharge voltage and current agrees very well with the measurement result. 


\section{Acknowledgments}

This work was supported by the "German Research Foundation" (DFG) in the frame of the "Reaserch Unit FOR 1123 - Physics of Microplasmas" and the Research School of the Ruhr-University Bochum. The authors would also like to thank Bernd Becker, Frank Kremer and Thomas Zierow for the technical support.

\section{References}

1 K. H. Schoenbach, R. Verhapen, T. Tessnow, F. E. Peterkin and W. W. Byszwski, Appl. Phys. Lett. 68 (1996) 13-15.

2 A. El-Habachi and K. H. Schoenbach, Appl. Phys. Lett. 73 (1998) 885-887.

3 A. El-Habachi, W. Shi, M. Moselhy, R. H. Stark and K. H. Schoenbach, J. Appl. Phys. 88 (2000) 3220-3224.

4 K. H. Schoenbach, A. El-Habachi, M. Moselhy, W. Shi and R. H. Stark, Phys. Plasmas 7 (2000) 2186-2191.

5 M. Moselhy, W. Shi, R. H. Stark and K. H. Schoenbach, Appl. Phys. Lett. 79 (2001) 1240-1242.

6 I. Petzenhauser, L. D. Biborosch, U. Ernst, K. Frank and K. H. Schoenbach, Appl. Phys. Lett. 83 (2003) 4297-4299.

7 F. Leipold, R. H. Stark, A. El-Habachi and K. H. Schoenbach, J. Phys. D: Appl. Phys. 33 (2000) 2268-2273.

8 M. Miclea, K. Kunze, U. Heitmann, S. Florek, J. Franzke and K. Niemax, J. Phys. D: Appl. Phys. 38 (2005) 1709-1715.

9 K. H. Schoenbach, A. El-Habachi, W. Shi and M. Ciocca, Plasma Sources Sci. Technol. 6 (1997) 468-477.

10 D. D. Hsu and D. B. Graves, J. Phys. D: Appl. Phys. 36 (2003) 2898-1907.

11 A. Rousseau and X. Aubert, J. Phys. D: Appl. Phys.39 (2006) 1619-1622.

12 X. Aubert, G. Bauville, J. Guillon, B. Lacour, V. Puech and A. Rousseau, Plasma Sources Sci. Technol. 16 (2007) 23-32.

13 K. H. Becker, K. H. Schoenbach and I. G. Eden, J. Phys. D: Appl. Phys. 39 (2006) R55-R70.

14 M. Moselhy, I. Petzenhauser, K. Frank and K. H. Schoenbach, J. Phys. D: Appl. Phys. 36 (2003) 2922-2927.

15 Q. Wang, I. Koleva, V. M. Donnelly and D. J. Economou, J. Phys. D: Appl. Phys. 38 (2005) 16901697.

16 A. Yanguas-Gil, K. Focke, J. Benedikt and A. von Keudell, J. Appl. Phys. 101 (2007) 103307.

17 X. Zhu, W. Chen and Y. Pu, J. Phys. D: Appl. Phys. 41 (2008) 105212.

18 H. -J. Kunze, Introduction to Plasma Spectroscopy (2009), Springer Heidelberg.

19 Gordon W F Drake, Atomic, Molecular, \& Optical Physics Handbook, American Institute of Physics, Woodbury, New York.

20 M. A. Gigosos, M. Á. González and V. Cardeñoso, Spectrochimica Acta Part B 58 (2003) 14891504.

21 D. D. Hsu and D.B. Graves, J. Phys. D: Appl. Phys. 36 (2003) 2898-2907.

22 A. Fridman and L. A. Kennedy, Plasma Physics and Engineering, Taylor \& Francis Group, 2004 